\title{
Toward a Scoring Function for Quality-Driven Machine Translation
}

\author{
Douglas A. Jones 1 \\ Department of Defense \\ 9800 Savage Road, Suite 6514 \\ Fort Meade, MD 20755-6514
}

\author{
Gregory M. Rusk \\ RABA Technologies \\ 10500 Little Patuxent Parkway \\ Columbia, MD 21044
}

\begin{abstract}
We describe how we constructed an automatic scoring function for machine translation quality; this function makes use of arbitrarily many pieces of natural language processing software that has been designed to process English language text. By machine-learning values of functions available inside the software and by constructing functions that yicld values based upon the software output, we are able to achieve preliminary, positive results in machine-learning the difference between human-produced English and machine-translation English. We suggest how the scoring function may be used for MT system development.
\end{abstract}

\section{Introduction to the MT Plateau}

We believe it is fair to say that the field of machine translation has been on a plateau for at least the past decade. ${ }^{2}$ Traditional, hand-built MT systems held up very well in the ARPA MT evaluation (White and O'Connell 1994). These systems are relatively expensive to build and generally require a trained staff working for several years to produce a mature system. This is the current commercial state of the art: hand-building specialized lexicons and translation rules. A completely different type of system was competitive in this evaluation, namely, the purely statistical CANDIDE system built at IBM. It was generally felt that this system had also reached a plateau in that more data and more training was not likely to improve the quality of the output.

\section{Low Density Machine Translation}

However, in the case of "Low Density Machine Translation" (see Nirenburg and Raskin 1998, Jones and Havrilla 1998) commercial market forces are not likely to provide significant incentives for machine translation systems for Low Density (Non-Major) languages any time soon. Two noteworthy efforts to break past the data and labor bottlenecks for high-quality machine translation development are the following. The NSF Summer Workshop on

1 Douglas Jones is now at National Institute of Standards \& Technology, Gaithersburg, MD 20899, Douglas.Jones@NIST.gov

2 A sensible, plateau-friendly strategy may be to accumulate translation memory to improve both the long-term efficiency of human translators and the quality of machine translation systems. If we imagine that the plateau is really a kind of logarithmic function tending ever upwards, we need only be paticnt.
Statistical Machine Translation held at Johns Hopkins University summer 1999 developed a public-domain version intended as a platform for further development of a CANDIDE-style MT system. Part of the goal here is to improve the translation by adding levels of linguistic analysis beyond the word N-gram. An effort addressing the labor bottleneck is the Expedition Project at New Mexico State University where a preliminary elicitation environment for a computational field linguistics system has been developed (the Boas interface; see Nirenburg and Raskin 1998)

\section{A Scoring Function for MT quality}

Our contribution toward working beyond this plateau is to look for a way to define a scoring function for the quality of the English output such that we can use it to machine-learn a good translation grammar. The novelty of our idea for this function is that we do not have to define the internals of it ourselves per se. We are able to define a successful function for two reasons. First, there is a growing body of software worldwide that has been designed to consume English; all we need is for each piece of software to provide a metric as to how Englishlike its input is. Second, we can tell whether the software had trouble with the input, either by system-internal diagnosis or by diagnosing the software's output. A good illustration is the facility in current word-processing software to put red squiggly lines underneath text it thinks should be revised. We know from experience that this feature is often only annoying. Nevertheless, imagine that it is correct some percentage of the time, and that each piece of software we use for this purpose is correct some percentage of the time. Our strategy is to 
extract or create numeric values from each piece of software that corresponds to the degree to which the software was happy with the input. That array of numbers is the heart of our scoring function for Englishness -. we are calling these numeric values "indicators" of Englishness. We then use that array of indicators to drive the machine translation development. In this paper we will report on how we have constructed a prototype of this function; in separate work we discuss how to insert this function into a machine-learning regimen designed to maximize the overall quality of the machine translation output.

\section{A Reverse Turing Test}

People can generally tell the difference between human-produced English and machine translation English, assuming all the obvious constraints such as that the reader and writer have command of the language. Whether or not a machine can tell the difference depends of course, on how good the MT system is. Can we get a machine to tell the difference? Of course it depends on how good the MT system is: if it were perfect, neither we nor the machines ought to be able to distinguish them. MT quality being what it is, that is not a problem for us now. An essential first step toward QIDMT is what we are calling a "Reverse Turing Test". In the ordinary Turing "Test, we want to fool a person into thinking the machine is a person. Here, we are turning that on its head. We want to define a function that can tell the difference between English that a human being has produced versus English that the machine has produced." To construct the test, we use a bilingual parallel aligned corpus: we take the foreign language side and send that through the MT system; then we see if we can define a scoring function that can distinguish the two versions (original English and MT English). With our current indicators and corpus, we can machine-learn a function that behaves as follows: if you hand it a human sentence, it correctly classifies it as human $74 \%$ of the time. If you hand it a machine sentence, it correctly classifies it as a machine sentence $57 \%$ of the time. In the remainder of the paper, we will step through the details of the experiment; we will also discuss why we

3Obviously the end goal here is to fail this Reverse Turing l'est for a "perfect" machine translation system. We are very far away from this, but we would like to use this function to drive the process toward that eventual and fortunate failure. neither expect nor require $100 \%$ accuracy for this function. Our boundary tests behave as expected and are shown in the final section -we use the same test to distinguish between English and (a) English word salad, (b) English alphabet soup, (c) Japanese, and (d) the identity case of more human-produced English.

\section{Case Study: Japanese-English}

In this paper, we report on results using a small corpus of 2,340 sentences drawn from the Kenkyusha New Japanese-English Dictionary. It was important in this particular experiment to use a very clean corpus (perfectly aligned and minimally formatted). This case study is situated in a broader context: we have conducted exploratory experiments on samples from several corpora, for example the ARPA MT Evaluation corpus, samples from European Corpus Initiative Data corpus (ECl-1) and others. Since we found that the scoring function was quite sensitive to formatting problems (for example, the presence of tables and sentence segmentation errors cause problems) we are examining a small corpus that is free from these issues. The sentences are on average relatively short $(7.0$ words per sentence; 37.6 characters/sentence), this makes our task both easier and harder. It is easier because we have overcome the formatting problems. It is harder because the MT system is able to perform much better on the shorter, cleaner sentences than it was on longer sentences with formatting problems. Since the output is better, it is more difficult to define a function that can tell the difference between the original English and the machine translation English. On balance, this corpus is a good one to illustrate our technique.

\begin{tabular}{|c|c|c|}
\hline \multirow[t]{3}{*}{ (1) } & \#208 & 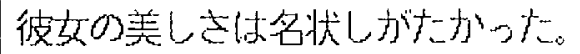 \\
\hline & & Her beatuty baffled description \\
\hline & $\mathrm{MT}$ & $\begin{array}{l}\text { It described her beatuty and the abnormal } \\
\text { play applied }\end{array}$ \\
\hline \multirow[t]{3}{*}{$(2)$} & $\# 1577$ & 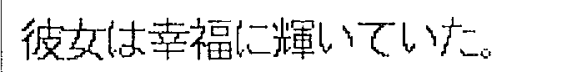 \\
\hline & & She was radiant with happiness \\
\hline & MT & She had shone happily \\
\hline \multirow[t]{3}{*}{ (3) } & $\# 1802$ & 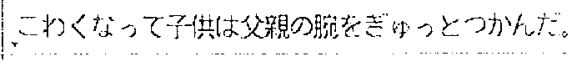 \\
\hline & & $\begin{array}{l}\text { In terror the child seized his father's } \\
\text { arm. }\end{array}$ \\
\hline & $\mathrm{MT}$ & $\begin{array}{l}\text { Becoming fearful, the child } \\
\text { grasped the arm of the father tightly }\end{array}$ \\
\hline
\end{tabular}

Figure 1. Subjective Quality Ranking 
Figure 1 shows a range of output quality. (1) is the worst -- it is obviously MT output. For us this output is only partially intelligible. (2) is not so bad, but it is still not perfect English. But (3)is nearly perfect. We want to design a system that can tell the difference. We will now walk through our suite of indicators; the goal is to get the machine to see what we see in terms of quality.

\section{Suite of Indicators}

We have defined a suite of functions that operate at various levels of linguistic analysis: syntactic, semantic, and phonological (orthographic). For each of these levels, we have integrated at least one tool for which we construct an indicator function. The task is to use these indicators to generate an array of values which we can use to capture the subjective quality we see when we read the sentences. We will step through these indicator functions one by one. In some cases, in order to get numbers, we take what amounts to debugging information from the tool (many of the tools have very nice API's that give access to a variety of information about how it processed input). In other cases, we define a function that yields an output based on the output of the tool (for example, we defined a function that indicated the degree to which a parse tree was balanced; it turned out that a balanced tree was a negative indicator of Englishness, probably because English is rightbranching).

\section{Syntactic Indicators}

Two sources of local syntactic information are (a) parse trees and (b) N-grams. Within the parsers, we looked at internal processing information as well as output structures. For example, we measured the probability of a parse and number of edges in the parse from the Collins parser. The Apple Pie Parser provided various weights which we used. The Appendix lists all of the indicator functions that we used.

\section{$N$-Gram Language Model (Cross-Perplexity)}

An easy number to calculate is the crossperplexity of a given text, as calculated using an N-gram language model. ${ }^{4}$

\footnotetext{
${ }^{4}$ We used the Cambridge/CMU language modeling toolkit, trained on the Wall Street Journal (4/1990 through 3/1992), (Im parameters: $n=4$, Good-Turing smoothing)
}

\begin{tabular}{|c|c|c|}
\hline & $\begin{array}{l}\text { Cross- } \\
\text { perplexity }\end{array}$ & \\
\hline (1) & 2439 & $\begin{array}{l}\text { It described her beauty and the } \\
\text { abnormal play applied }\end{array}$ \\
\hline$(2)$ & 2185 & She had shone happily \\
\hline (3) & 1836 & $\begin{array}{l}\text { Becoming fearful, the child } \\
\text { grasped the arm of the father } \\
\text { tightly }\end{array}$ \\
\hline
\end{tabular}

Notice that the subjective order is mirrored by the cross-perplexity scores in Figure 2.

\section{Collins Parser}

The task here is to write functions that process the parse trees and return a number. We have experimented with more elaborate functions that indicate how balanced the parse tree is and less complicated functions such as the level of embedding, number of parentheses, and so on. Interestingly, the number of parentheses in the parse was a helpful indicator in conjunction with other indicators.

\section{Indicators of Semantic Cohesiveness}

For the semantic indicators, we want some indication as to how much the words in a text are related to each other by virtue of their meaning. Which words belong together, regardless of exactly how they are used in the sentence? Two resources we have begun to integrate for this purpose are WordNet and the Trigger Toolkit (measuring mutual information). The overall experimental design is roughly the same in both cases. Our method was to remove stop words, lemmatize the text, and then take a measurement of pairwise semantic cohesiveness of the lemmatized words $s^{5}$. For WordNet, we are counting how many ways two words are related by the hyponymy relation (future indicators will be more sophisticated). For the Trigger Toolkit, we weighted the connections (by mutual information).

\section{Orthographic}

We had two motivations for an orthographic level: one was methodological (we wanted to look at cach of the traditional levels of linguistic analysis). The other was driven by our experience in looking at the MT output. Some MT systems leave untranslated words

\footnotetext{
${ }^{5}$ The following parameters were used to build and calculate mutual information using the Trigger Toolkit: (1) All uppercase letters were converted to lowercase (2) All numbers were converted to a "NUMBER" token (3) Punctuation stripped (4) Stopwords removed (5) Words lemmatized.
} 
alone, or transliterate them, or insert a dummy symbol, such as "X". These clues were adequate to give us appropriate hints as to whether the text was produced by human or by machine. But some of our tools missed these clues because of how they were designed. Robust parsers often treat unknown words as nouns; so if we get an untranslated term or an " $X$ ", the parser simply treats it as a noun. live $X$ 's in a row might be a noun phrase followed by a verb. ${ }^{6}$ Smoothed N-gram models of words usually treat any string of letters as a possible word.

\begin{tabular}{|c|c|c|c|c|c|}
\hline $\begin{array}{l}\text { MT } \\
\text { output }\end{array}$ & $\begin{array}{l}\text { Word N- } \\
\text { gram } \\
\text { Cross Per- } \\
\text { plexily }\end{array}$ & $\begin{array}{l}\text { Num. } \\
\text { Ldges }\end{array}$ & $\begin{array}{l}\text { Apple } \\
\text { Pic } \\
\text { Parser } \\
\text { Score }\end{array}$ & $\begin{array}{l}\text { Sumof } \\
\text { mutual } \\
\text { infor-- } \\
\text { mation }\end{array}$ & $\begin{array}{l}\text { Char } \\
\text { N-gram } \\
\text { cross } \\
\text { per- } \\
\text { plexity }\end{array}$ \\
\hline $\begin{array}{l}\text { worst } \\
\text { (1) }\end{array}$ & 2439 & 152 & 247 & 0 & 8.1 \\
\hline $\begin{array}{l}\text { mid } \\
(2)\end{array}$ & 2185 & 271 & 139 & 0 & 16.3 \\
\hline $\begin{array}{l}\text { best } \\
(3)\end{array}$ & 1836 & 1654 & 302 & $1.7 \mathrm{~F}-4$ & 9.3 \\
\hline
\end{tabular}

Figure 3. Subjective and Objective Rankings

Because the parsers and N-gram models were designed to be very robust, they are not necessarily sensitive to these obvious clues. In order to get at these hints, we built a characterbased N-gram model of English. Although these indicators were not very informative on their own for distinguishing human from machine English, they boosted performance in conjunction with the syntactic and semantic indicators.

\section{Combined Indicators}

Let's come back to the three sentences from Figure 1: we want to capture our subjective ranking of the sentences with appropriate indicator values. In other words, we want the machine to be able to see differences which a human might see.

For these three examples, some scores correlate well with our subjective ranking of Englishness (e.g. cross-perplexity, Edges). However, the other scores on their own only partially correlate. The expectation is that an indicator on its own will not be sufficient to score the Englishness. It is the combined effect of all indicators which ultimately decides the

"We found that we could often guess the "default" behavior that a parser used and we have begun to design indicators that can tell when a parser has defaulted to these.
Englishness. Now we have enough raw data to begin machine-learning a way to distinguish these kinds of sentences.

\section{Simple Machine Learning Regimen}

We have started out with very simple memorybased machine lcarning techniques. Since we are defining a range of functions, we wanted to keep things relatively simple for debugging and didactic purposes.

\section{KNN}

One of the simplest methods we can use for classification is to collect values of the $\mathrm{N}$ indicators for a set of training cases and for the test cases, to find the $\mathrm{K}$ nearest training cases (using Fuclidean distance in $\mathrm{N}$-dimensional space). For $\mathrm{K}$, we used 5 for our general experiments (but see below for some variations). For a concrete example in two dimensions, imagine that we use the crossperplexity of an $\mathrm{N}$-gram language model for the $Y$-axis and the probability of a parse from the Collins parser for the X-axis. Human sentences tended to have better (lower) cross-perplexity numbers and better (higher) parse probabilities. If the 5 nearest neighbors to a data point were $(h, h, h, h, m)$ four human sentences and one machine our KNN function guesses that it is a human sentence.

Figure 4 lists some of the parameters we used for KNN. The values for cross perplexity ranged from around 100 to 10,000 and the Collins parse probability (log) ranged from around -1000 to 0 . These values were normalized to range from $0-1$.

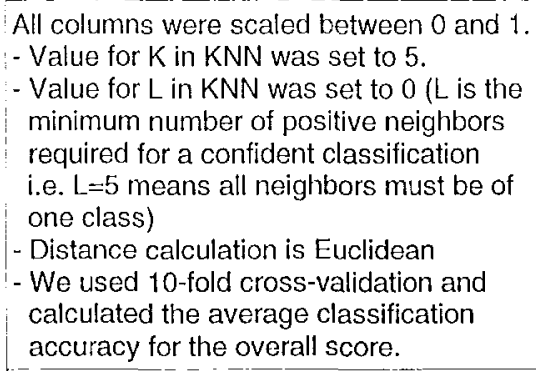

Figure 4. KNN Parameters

To get an indication of how much guessing figured into the classification, we varied $\mathrm{L}_{-}$from 3 to 5 , keeping $\mathrm{K}$ at 5 . We found that we get the same overall shape for the classification, with fewer guesses made. Of course the penalty for not guessing as much is that more cases are left unclassified. When we reduced guessing by setting $L$ to 4 , we correctly classified $47 \%$ of the human sentences as human and incorrectly classified $9 \%$ of the human sentences as 
machine (the remaining $44 \%$ were not classified). By setting $\mathrm{L}$ to 5 (eliminating guessing) these numbers dropped to $18 \%$ and $2 \%$ respectively. When we varied $\mathrm{K}$ (for example, trying $\mathrm{K}$ of 101) we found that we can increase the performance of the human classifier to nearly $90 \%$. Performance of $\mathrm{KNN}$ tended to top out at around $74 \%$ with the parameters in Figure 4.

\section{Indicator Monotonicity}

There is no guarantee that classification will perform better with more dimensions in KNN. However, we found that we generally got a monotonically increasing performance in classification when we added indicator functions. A helpful analogy might be to consider the blind men and the elephant. In our case, "English" is the elephant, and each of our indicator functions is one blind man grasping at the elephant. One is grasping at semantics, one at syntax, and so on. Figure 5 shows how classification improves with more indicators (the back of the elephant, so to speak).

\section{Benchmarks}

To calibrate the indicator functions we have used to classify text into human- or machineproduced, we tested our method with some boundary cases, shown in Figure 6. The most extreme case was to learn the difference

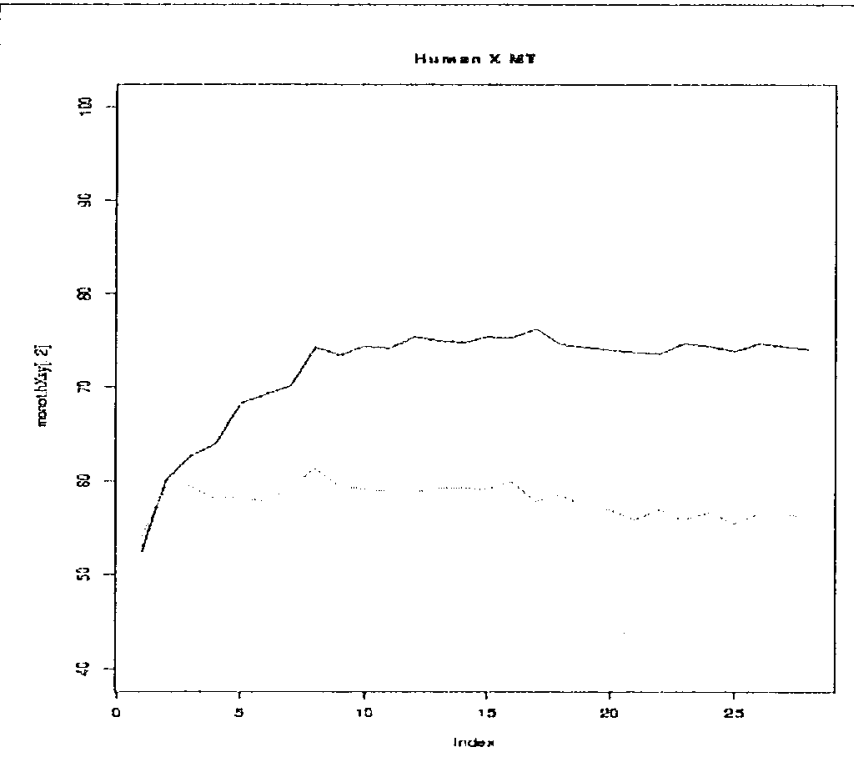

Top: Truth is human; machine guesses human

Bottom: Truth is MT; machine guesses MT

Figure 5

between Japanese text (in native character encoding) and English.

\begin{tabular}{|l|l|l|}
\hline & Truth is: & \\
\hline & human & machine \\
\hline $\begin{array}{l}\text { Machine } \\
\text { Guesses: }\end{array}$ & & \\
\hline Japanese & 99.6 & 99.6 \\
\hline Alphabet Soup & 99.4 & 99.2 \\
\hline Word Salad & 95.4 & 91.1 \\
\hline MT Output & 74.0 & 56.1 \\
\hline Identity Case & 52.3 & 49.4 \\
\hline
\end{tabular}

Figure 6. Baselines

In other words, we have come up with a very computationally expensive method for Language Identification. Next less extreme was what we called "Alphabet Soup"; we took English sentences from the English side of the Kenkyusha corpus: for each alphabetic character, we substituted a randomly-selected alphabetic character, preserving case and punctuation." For "Word Salad", we took the English sentences from the Kenkyusha corpus and scrambled their order. MT Output is the case we discussed in detail above. The Identity Case is to divide the English sentences from the corpus into two piles and then try to tell them apart. As Figure 6 shows, the pathological baseline cases all work out very well: our machine can almost always tell that Japanese, Alphabet Soup, and Word Salad are not English. Nor can it distinguish between two arbitrarily divided piles of human English.

\section{Other Classification Algorithms}

We have performed some initial experiments with Support Vector Machines (SVM) as a classification method. SVM attempts to divide up an ndimensional space using a set of support vectors defining a hyperplane. The basic approach of the SVM algorithm is different from $\mathrm{KNN}$ in that it actually deduces a classification model from the training data. KNN is a memory-based learning algorithm wherein the model is essentially a replica of the training examples.

The initial trials using SVM are yielding classification accuracies of correctly classifying $83 \%$ of the human sentences and $64 \%$ of the machine sentences (single

${ }^{7} \mathrm{We}$ found that it was often easy to crash some of the software when we fed it arbitrary binary data, so we used "Alphabet Soup" instead of arbitrary binary data. 
random sample of $10 \%$ withheld -- no n-fold cross-validation). These accuracies represent improvements of $11 \%$ for human test sentences and $14 \%$ for the machine test sentences. Further tests on this and other classification methods will be investigated to maximize performance in terms of accuracy and execution time.

\section{Next Steps}

There are two general areas we are continuing to work on: (a) to increase the scope and reliability of our indicators and (b) to insert the scoring function into a machine-learning regimen for producing translation grammars. In the first area, we have begun to explore the degree 10 which we might recapitulate the ARPA MT Evaluation. The data from these evaluations are freely available. ${ }^{8}$ Of course if all we did was recapitulate the data in some non-explanatory way, we would be doing something analogous to using the Chicago Bears to predict the stock market. The real work here is to map the objective scoring function numbers back to reliable subjective evaluation of the machine-produced texts. A crucial task for us here is to get a deeper understanding of how each of the pieces of software behaves with various types of input text. We are currently at a quite preliminary stage in terms of the number of indicators we are using and the degree to which each is finetuned to our purpose. For machine-learning a translation grammar, we have begun to explore using our scoring function to drive the construction of a prototype Low Density machine translation grammar compatible with a previous system built by hand. We have found that the scoring function is sensitive to the word order difference between the target English translation and the glosses for the source language. We would like to re-create a compatible knowledge base of the English half of the translation grammar using only the glosses as input. Such a technique would reduce the labor requirements in constructing a translation knowledge base.

\section{Reverse Turing Scores for Machine Learning Grammars}

To illustrate how we can use the Reverse Turing scores to machine learn a grammar, let us consider a simple case of learning lexical features for a unification-based phrase structure grammar of the sort discussed in Jones \&

${ }^{8}$ From ursula.georgetown.edu/mt_web.
Havrilla 1998. The working assumption there is that an adequate translation grammar can be created that conforms to the constraint that the only reordering that is allowed is the permutation of nodes in a binary-branching trec (as in Wu 1995, among others). How might we learn that postpositions and verbs generally trigger inversion? Consider the following example as shown in Figure 7 from Jones \& Havrilla 1998; let us use $+\mathrm{T}$ to indicate that the lexical item triggers inversion; - $\mathrm{T}$ means that it does not. Let the initial state of the lexicon mark all lexical items as "-T".

\begin{tabular}{|l|l|l|l|l|}
\hline $\mathrm{S}$ & Shobhaa & kamre-men & baiThii & hai \\
\hline POS & $\mathrm{N}$ & $\mathrm{N} \mathrm{O}$ & $\mathrm{V}$ & $\mathrm{V}$ \\
\hline $\mathrm{F}$ & $-\mathrm{T}$ & $-\mathrm{T}+\mathrm{T}$ & $+\mathrm{T}$ & $+\mathrm{T}$ \\
\hline & Shobha & the_room-in & sitting & is \\
\hline
\end{tabular}

Figure 7. Shobha is sitting in the room.

Our machine learning process marks lexical items as " $+\mathrm{T}$ " when the Reverse Turing classification score for the bilingual corpus improves.

\section{Conclusion}

We are capitalizing on two historical accidents: (1) that English is a major world language and (2) that we want to translate into English. In addition to a variety of modern, standard NLP techniques and ideas, we have drawn from two unlikely sources of intellectual capital: (1) philosophy of language and (2) the current ubiquity of language engineering software. What we have taken from (1) is that we have assumed that there is such a thing as "English". That might not seem like much of an assumption, but we are treading near some very thorny problems in the philosophy of language. We can no more point to English than we can point to the perfect triangle. And like the blind men grasping at the elephant, how we characterize it depends on how we are exploring it. What is important is the helpful aggregate of numeric values that we use for the scoring. What does this mean for machine translation? We want to "Begin with the End in Mind"; in other words, we want the machine translation system to create output that scores well on our indicators of Englishness. The rest would be details, so to speak.

\section{Acknowledgments}

This project was funded in part by the Advanced Research and Development Activity. We would like to thank our colleagues at DoD for very helpful discussions and insights. 


\section{Appendix}

List of current Indicators

1. Word N-Gram (CMU/Canbridge Language Tk)

2. Number of edges in parse (Collins Parser)

3. Log probability (Collins Parser)

4. Execution time (Collins Parser)

5. Paren count (Collins Parser)

6. Mean lcaf node size of parse tree (Collins Parser)

7. Mean NN sequence length (Collins Parser)

8. Overall score (Apple Pie Parser)

9. Word lcvel score (Apple Pic Parser)

10. Node count (Apple Pie Parser)

11. User execution time (Apple Pie Parser)

12. CD node count (Apple Pie Parser)

13. Mean CD sequence length (Apple Pie P'arser)

14. Mean leading spaces in outline tree (from Collins Parse)

15. Tree balance ratio (from Collins Parse)

16. Tree depth (from Collins Parse)

17. Average minimum hypernym path length in WordNet

18. Average number hypernym paths in WordNet

19. Path found ratio in WordNet

20. Pereent words with sense in WordNer

21. Sum of count of relations (Trigger Toolkit)

22. Mean of count of relations (Trigger Toolkit)

23. Sum of mutual information (Trigger Toolki1)

24. Mean of mutual information (Trigger Toolkit)

25. Pairs with mutual information (Trigger Toolkit)

26. Weighted pair sum of mutual information (Trigger Toolkit)

27. Number of target paired words (Trigger Toolkit

27. . N-Gram Cross-perplexity (Cambridge/CMU Lang Tk.)

\section{Tools}

TiMBL: Tilburg Memory Based Learner 2.0. ILK Research Group. http://ilk.kub.nl/software.html.

PCKIMMO 2.0. Summer Institute of Linguistics.

MXTERMINATOR. Adwait Ratnaparkhi.

WEKA 3.0. University of Waikato. [tp://lp.cs.waikato.ac.nz/pub/ml/weka-3-0.jar

Collins Parser 98.

Brill Tagger 1.14

R Statistical Package 0.65.0. http://cran.rproject.org/

Apple Pie Parser 5.8. New York University. http://cs.nyu.edu/cs/projects/proteus/app

WordNet 1.6. ftp://ftp.cogsci.princeton.edu/ wn/

Trigger Toolkit 1.0. CMU. http://www.cs.cmu.edu/ aberger/software.

\section{References}

Afifi, A.A., Virginia Clark. 1996. Computer-Aided Multivariate Analysis, 3rd ed.. New York, NY: Chapman and Hall.

Brill, Eric. 1995. Transformation-Based Error-

Driven Learning and Natural Language

Processing: A Case Study in Part Of Specch

Tagging. (ACL).

Chambers, John M., William S. Cleveland, Beat

Kleiner, and Paul A. Tukey. 1983. Graphical

Methods for Data Analysis. Boston, MA: Duxbury

Press.

Clarkson, Philip, Ronald Rosenfeld. 1997.

Statistical Language Modeling Using the CMU-

Cambridge Toolkit. Eurospeech97. Rhodes,

Greece

Collins, Michael. 1997. Three Generative, Lexicalised Models for Statistical Parsing. Proceedings of the 35th Annual Mecting of the ACL/EACL), Madrid.
Daclemans, Walter, Jakub Zavrel and Ko van der Sloot. 1998. TiMBL: Tilburg Memory Based Learner, version 2.0, Reference Guide. Available from http://ilk.kub.nl/softwarc.html.

Everitt, Brian S. and Graham Dunn. 1992. Applied Multivariatc Data Analysis. New York, NY: Oxford University Press.

Fellbaum, Christiane (ed.). 1998. WordNet: An Electronic Lexical Database. Cambridge, MA: The MIT Press.

Flury, Bernhard and Hans Riedwyl. 1988. Multivariate Statistics. New York, NY: Chapman and Hall.

Hornik, Kurt. 1999. "The R FAQ". Available at http://www.ci.luwicn.ac.at/ hornik/R/.

Jones, Doug and Rick Havrilla. 1998. Twisted Pair Grammar: Support for Rapid Development of Machine Translation for Low Density Languages. AMTA-98. Langhorn, PA.

Knight, Kevin, Ishwar Chandler, Matthew Haines, Vasileios, Hatzivassigloglou, Eduard Hovy, Masayo Ida, Steve Luk, Richard, Whitney, and Kenji Yamada. 1994. Integrating Knowledge Bases and Statistics in MT (AMTA-94)

Manning, Christopher D. and Hinrich Schutze. 1999. Foundations of Statistical Natural Language Processing. Cambridge, MA: The MIT Press.

Masuda, Koh (ed). 1974. Kenkyusha's New Japanese-English Dictionary, 4th Ed. Tokyo: Kenkyusha.

Michalski, Ryszard S., Ivan Bratko, and Miroslav Kubat (cds.). 1998. Machine Learning and Data Mining. John Wilcy \& Son.

Mitchell, Tom M. 1997. Machine Learning. Boston, MA: McGraw-Hill.

Nirenburg, S. and V. Raskin. 1998. Universal Grammar and Lexis for Quick Ramp-Up of MT Systems. Procecdings of ACL/COLING ` 98. Montréal: University of Montreal (in press).

Reynar, Jeffrey C. and Adwait Ratnaparkhi. 1997. A Maximum Entropy Approach to Identifying Sentence Boundaries. ANLP-97. Washington, D.C.

Rosenfeld, Ronald. 1996. A Maximum Entropy Approach to Adaptive Statistical Language Modeling. Computer, Specch and Language.

Witten, Ian H. and Eibe Frank. 1999. Data Mining: Practical Machine Learning Tools and Techniques with Java Implementations. Morgan Kaufmann.

White, J. and T.A. O'Connell. 1994. The ARPA MT Evaluation Methodologies: Evolution, Lessons, and Future Approaches. Procecdings of AMTA94

Wu, Dekai and Xuanyin Xia. 1995. Large-Scale Automatic Extraction of an English-Chinese Translation Lexicon. Machine Tranlsation. 9:3, 128. 